J. Lake Sci. (湖泊科学) , 2021, 33(6): 1820-1831

DOI 10. $18307 / 2021.0620$

(C) 2021 by Journal of Lake Sciences

\title{
红河 (元江) 流域的典型鱼类游泳能力探究及在过鱼设施流速设计中 的应用
}

\author{
王永猛 ${ }^{1,2}$,柯森繁 ${ }^{1,2}$, 林晨宇 ${ }^{3}$, 张 奔 $^{1,2}$,杨国党 ${ }^{1,2}$, 雷青松 ${ }^{4}$, 邓晓川 ${ }^{5}$, 石小涛 ${ }^{1,2 * *}$ \\ (1:三峡大学水利与环境学院,宜昌 443002) \\ (2:湖北省鱼类过坝技术国际科技合作基地,宜昌 443002) \\ (3: 河海大学水利水电学院, 南京 210098$)$ \\ (4:广州市天河区水务设施建设中心,广州 510655) \\ (5: 山东省水利勘测设计院, 济南 250000$)$
}

\begin{abstract}
摘 要: 为探讨红河 (元江) 流域的典型洄游鱼类游泳能力, 给过鱼设施修建设计和鱼类行为学研究提供基础参数, 本研 究以该流域的野生暗色唇鲮(Semilabeo obscurus)、华南鲤(Cyprinus carpio rubrofuscus) 和红鮕 (Bagarius rutilus) 3 种鱼类(体 长范围 0.05 0.45 m) 为研究对象, 在野外现场布置游泳能力测试水槽分别测试了感应流速、临界游泳速度和突进游泳速 度. 研究结果表明: (1) 对比绝对游泳能力与体长相关性, 3 种鱼的相对游泳能力与体长的相关性更高, 均呈显著负相关; (2) 3 种鱼之间的游泳能力存在差异, 红鮕的感应流速最大, 为 $(0.10 \pm 0.01) \mathrm{m} / \mathrm{s}$, 华南鲤的临界游泳速度最大, 为 $(0.66 \pm$ $0.01) \mathrm{m} / \mathrm{s}$, 华南鲤的突进游泳速度最大,为 $(1.48 \pm 0.18) \mathrm{m} / \mathrm{s}$; (3) 拟合趋流率与水流速度的相关曲线,所有测试鱼的趋流 率与流速的函数关系为 $P r e=19.80 \mathrm{~V}-1.13\left(R^{2}=0.96, P<0.05\right)$, 得出 $95 \%$ 目标鱼的趋流速度为 $0.11 \mathrm{~m} / \mathrm{s}$; 利用生存分析方 法, 得出 $95 \%$ 目标鱼累积疲劳的临界和突进游泳速度分别为 $0.58 、 1.01 \mathrm{~m} / \mathrm{s}$. (4) 当以暗色唇鲮、华南鲤和红鮕为主要过鱼 对象时,建议过鱼设施的内部流速不应小于 $0.1 \mathrm{~m} / \mathrm{s}$, 人口的设计流速范围为 $0.6 \sim 1.0 \mathrm{~m} / \mathrm{s}$, 休息区域的流速范围为 $0.1 \sim$ $0.6 \mathrm{~m} / \mathrm{s}$, 孔口或坚缝等高流速区的设计流速不宜超过 $0.8 \mathrm{~m} / \mathrm{s}$, 若过鱼设施孔口边壁或底部加楉, 可适当增至 $0.8 \sim 1.0 \mathrm{~m} / \mathrm{s}$. 本研究中红河 (元江) 流域鱼类游泳能力等资料为过鱼设施修建及珍稀鱼类保护提供了数据支撑.
\end{abstract}

关键词: 暗色唇鲮;华南鲤;红鮕; 游泳能力;过鱼设施;生存分析;红河 (元江) 流域

\section{Investigation of the swimming ability of typical fish in the Red River(Yuanjiang) basin and its application to the design of fish passage facilities*}

\author{
Wang Yongmeng ${ }^{1,2}$, Ke Senfan ${ }^{1,2}$, Lin Chenyu ${ }^{3}$, Zhang Ben ${ }^{1,2}$, Yang Guodang ${ }^{1,2}$, Lei Qingsong ${ }^{4}$, Deng Xiao- \\ $\operatorname{chuan}^{5}$ \& Shi Xiaotao ${ }^{1,2 * *}$ \\ (1: College of Hydraulic and Environmental Engineering, China Three Gorges University, Yichang 443002, P.R. China) \\ (2: Hubei International Science and Technology Cooperation Base of Fish Passage, Yichang 443002, P.R.China) \\ (3: College of Water Conservancy and Hydropower Engineering, Hohai University, Nanjing 210098, P.R.China) \\ (4: Guangzhou Tianhe District Water Facilities Construction Center, Guangzhou 510655, P.R.China) \\ (5: Shandong Survey and Design Institute of Water Conservancy, Jinan 250000, P.R. China)
}

Abstract: To investigate the swimming capability of typical migrants and consequently serve the fishway design in the Red River (Yuanjiang) basin of China, we conducted an in-situ experiment on three presentative wild fish (body length: 0.05-0.45 m), i.e., the Semilabeo obscurus, Cyprinus carpio rubrofuscus, and Bagarius rutilus, testing their induced flow velocity, critical swimming speed, and burst swimming speed. The results indicated that (1) the relative swimming ability of the three species presented a signif-

* 2021-02-12 收稿;2021-03-30 收修改稿.

国家自然科学基金项目 $(51922065,51679126)$ 、湖北省高等学校优秀中青年科技创新团队计划项目 ( T201703) 和 三峡大学学位论文培优基金项目 (2020BSPY009)联合资助.

** 通信作者;E-mail: fishlab@163.com. 
icantly negative correlation with body length compared to the absolute swimming ability. (2) On the difference between species, the B. rutilus exhibited the highest inductive flow velocity of $(0.10 \pm 0.01) \mathrm{m} / \mathrm{s}$, while the $C$. carpio rubrofuscus ranked first at critical swimming velocity $((0.66 \pm 0.01) \mathrm{m} / \mathrm{s})$ and burst swimming velocity $((1.48 \pm 0.18) \mathrm{m} / \mathrm{s})$. (3) By fitting the percentage of rheotaxis and flow velocity, the functional relationship between them of all tested fish was Pre $=19.80 \mathrm{~V}-1.13\left(R^{2}=0.96, P<0.05\right)$, deriving the rheotaxis velocity of $95 \%$ target fish of $0.11 \mathrm{~m} / \mathrm{s}$. In survival analysis, the critical and burst swimming speeds of the $95 \%$ target fish were $0.58 \mathrm{~m} / \mathrm{s}$ and $1.01 \mathrm{~m} / \mathrm{s}$ for cumulative fatigue, respectively. (4) Altogether, the fish passages targeting $S$. obscurus, C. carpio rubrofuscus, and $B$. rutilus was suggested to generate the in-channel velocity larger than $0.1 \mathrm{~m} / \mathrm{s}$, the velocity at the entrance from 0.6 to $1.0 \mathrm{~m} / \mathrm{s}$, the main-flow velocity in rest pools between 0.1 to $0.6 \mathrm{~m} / \mathrm{s}$, and the high velocity at verticle slot and orifice less than $0.8 \mathrm{~m} / \mathrm{s}$ or slightly heighten to $0.8-1.0 \mathrm{~m} / \mathrm{s}$ with a rougher sidewall or bottom. Our study on fish swimming ability in the Red River (Yuanjiang) facilitates the construction of future fish passage and rare fish conservation.

Keywords: Semilabeo obscurus; Cyprinus carpio rubrofuscus; Bagarius rutilus; swimming ability; fish passage; survival analysis ; Red River ( Yuanjiang) basin

近年来, 为大力开发水能资源, 我国的水利工程建设数量逐年递增. 尤其在西南流域已建和规划了多个 梯级电站,如金沙江流域已建或规划的梯级电站达 20 余座 ${ }^{[1]}$. 梯级电站的修建在促进国计民生发展的同 时,对河流的生境也产生了不利影响, 其中因河流连通性被阻断引发的鱼类过坝困难的问题备受关注 ${ }^{[2]}$. 为 帮助鱼类洄游过坝, 过鱼设施 (鱼道、集运鱼船、升鱼机等) 近年来被大量修建, 据统计从 1958-2017 年, 我 国水利水电工程建设项目的已建和建设中的过鱼设施从 10 座增加至 150 余座 ${ }^{[3-5]}$.

过鱼设施的过鱼效果是直接反映恢复上下游物种交流及生态修复的重要依据 ${ }^{[6]}$. 影响过鱼设施过鱼效 果的因素有很多, 主要有生物因素和非生物因素, 生物因素包括鱼的体长、种类和体型等,非生物因素包括 流速、光和温度等 ${ }^{[7]}$. 影响鱼类游泳能力与行为的因素多为非生物因素, 非生物因素中的流场为关键因素, 具体有速度、矢量方向 (水流方向)、浴旋和紊动等, 其中流速是鱼类游泳能力可量化的关键指标 ${ }^{[8-10]}$. 目前 过鱼设施的流速设计主要参考过鱼对象的游泳能力阈值. 鱼类游泳能力通常包括感应流速、临界游泳速度 和突进游泳速度 ${ }^{[11]}$. 在过鱼设施设计中, 感应流速主要作为过鱼设施进口的最小设计流速 (吸引目标鱼的 水流速度) 参考值 ${ }^{[12]}$; 突进游泳速度是鱼类可达到的最大游泳速度, 主要用于过鱼设施高流速区 (坚缝) 的 流速设计 ${ }^{[13]}$; 临界游泳速度是鱼类最大可持续有氧运动能力指标 ${ }^{[13-14]}$, 主要用于过鱼设施内部低流速区域 的流速设计. 目前与鱼道设计相关的鱼类游泳能力研究大多聚集于单目标鱼种 ${ }^{[14-15]}$, 针对多目标鱼种的过 鱼设施设计参数选取的研究甚少. 本研究在过鱼设施设计参数选取过程中, 通过对游泳能力累积疲劳率与 流速的关系分析, 克服以往过鱼设施参数选取研究中的多目标和多参数的交互难度.

在进行过鱼设施流速设计时,所参考的过鱼对象往往不止一种,不同种的过鱼对象由于形态、生活习 性、栖息环境等不同,游泳能力会有明显差异 (比如上溯距离), 同一科不同种属的过鱼对象游泳能力也会有 差别. 比如, 同为鲉科的拟鳗副鲉 (Paracobits anguillioides) 和长薄鱾 (Leptobotia elongata) 突进游泳速度有显 著差异, 分别为 $(10.23 \pm 1.77)$ 和 $(6.10 \sim 18.50) \mathrm{BL} / \mathrm{s}^{[16]}$; 同为裂腹鱼亚科的灰裂腹鱼 (Schizothorax griseus) 和 光唇裂腹鱼 (Schizothorax lissolabiatus) 突进游泳速度分别为 $(6.36 \pm 1.12)$ 和 $(9.59 \pm 2.36) \mathrm{BL} / \mathrm{s}^{[17]}$. 基于不同 过鱼对象的游泳能力差异性, 鱼道进行流速设计时, 通常需要考虑弱游泳能力过鱼对象的过障需求. 因此, 针对不同大小和不同种类的过鱼对象开展现场游泳能力测试十分必要. 目前, 我国部分过鱼设施的流速设 计, 并没有对过鱼目标进行现场游泳能力测试, 尤其针对具有过坝需求的珍稀保护性洄游鱼类, 往往通过同 科不同种或者人工繁殖的相同鱼种的游泳能力进行替代. 然而, 用此方法所获取的游泳能力数据并不能真 实反映过鱼对象的速度过障能力, 如 Rouleau 等发现同一种类的野生美洲红点鲑比人工养殖的美洲红点鲑 游泳能力更强,并有显著的差异性 ${ }^{[18]}$.

马堵山水电站坝址位于西南地区红河 (元江) 干流红河哈尼族彝族自治州的个旧市和金平县境内, 是 《云南省红河 (元江) 干流综梯级合规划报告》的第 10 级开发电站, 也是开发方案中经济指标相对较好, 列为 先期开发建设的梯级之一. 马堵山水电站控制流域面积 $31356 \mathrm{~km}^{2}$, 多年平均流量 $299 \mathrm{~m}^{3} / \mathrm{s}$, 规划正常蓄水 位 $217 \mathrm{~m}$, 装机容量 $300 \mathrm{MW}$, 装机年利用时间为 $4600 \mathrm{~h}$, 年发电量 12.34 亿 $\mathrm{kW} \cdot \mathrm{h}$. 红河 (元江) 干流梯级电 站的逐步开发对红河 (元江) 流域的破碎化、土著鱼种洄游带来严重影响 ${ }^{[19]}$, 并且有关元江马堵山水电站至 
南沙水电站的上下游鱼类游泳能力研究成果较少. 因此, 本研究响应第 41 号环境评估报告要求 (中华人民 共和国环境保护部,2013 年), 依据马堵山水电站上下游的鱼类洄游习性,栖息地的适应性, 历史地位等主要 因素, 确定了暗色唇鲮、华南鲤和红魾三种为主要过鱼对象, 并在野外现场测试了感应流速、临界游泳速度 和突进游泳速度, 为该流域的过鱼设施流速设计提供重要的数据参考. 此研究对红河 (元江) 流域土著鱼类 和珍稀鱼类保护、栖息地修复和过鱼设施具有重要作用.

\section{1 材料与方法}

\section{1 研究区域与对象}

通过渔民走访调查和云南大学所承担的《云南省红河马堵山水电站工程施工期鱼类资源及陆生植被监 测报告》、珠江水资源保护科学研究所承担的《云南省红河马堵山水电站工程环境影响报告书》,统计出马堵 山水电站上下游水域出现鱼类 24 种. 过鱼对象主要依靠 5 个标准 (每种标准的等级是 $0 、 1 、 2$ ), 分别为洄游 习性、栖息地适应性、保护等级、经济价值、历史地位,每个等级具体评分见表 1. 如果每种鱼类的总等级大于 (等于) 7 ,那么此物种就被评为主要过鱼对象 ${ }^{[17]}$, 如表 2 所示.

表 1 过鱼对象选择标准 ${ }^{[20]}$

Tab.1 Criteria for target fish selection ${ }^{[20]}$

\begin{tabular}{|c|c|c|c|c|}
\hline & \multirow{2}{*}{ 标准 } & \multicolumn{3}{|c|}{ 等级评分 } \\
\hline & & 2 & 1 & 0 \\
\hline 通道要求 & 洄游习性 & 长距离洄游性 & 短距离洄游性 & 无洄游性 \\
\hline 通道利用 & 栖息地适应性 & $\begin{array}{c}\text { 高 } \\
\text { (原栖息地区仍然适应) }\end{array}$ & $\begin{array}{c}\text { 中 } \\
\text { (原栖息地区部分适应) }\end{array}$ & $\begin{array}{c}\text { 低 } \\
\text { (栖息地不再适应) }\end{array}$ \\
\hline 保护价值 & $\begin{array}{l}\text { 保护等级 } \\
\text { 经济价值 }\end{array}$ & $\begin{array}{c}\text { 国家级或物种濒临灭绝 } \\
\text { 高 }\end{array}$ & $\begin{array}{c}\text { 地方级或物种濒临灭绝 } \\
\text { 中 }\end{array}$ & $\begin{array}{c}\text { 未保护 } \\
\text { 低 }\end{array}$ \\
\hline 资源状况 & 历史地位 & 最近存在 & 以前存在 & 从未存在 \\
\hline
\end{tabular}

表 2 过鱼对象的确定

Tab.2 Determination of target fish based on criteria

\begin{tabular}{|c|c|c|c|c|c|c|c|c|c|}
\hline & & 中文名 & 拉丁名 & $\begin{array}{l}\text { 洄游 } \\
\text { 习性 }\end{array}$ & $\begin{array}{l}\text { 栖息地 } \\
\text { 适应性 }\end{array}$ & $\begin{array}{l}\text { 保护 } \\
\text { 等级 }\end{array}$ & $\begin{array}{l}\text { 经济 } \\
\text { 价值 }\end{array}$ & $\begin{array}{l}\text { 历史 } \\
\text { 地位 }\end{array}$ & 总 \\
\hline \multirow{7}{*}{$\begin{array}{c}\text { I 鲤形目 } \\
\text { Cypriniformes }\end{array}$} & $\mathrm{i}$ 鲤科 & 华南鲤 * & Cyprinus carpio rubrofuscus & 1 & 2 & 0 & 2 & 2 & 7 \\
\hline & Cyprinidae & 马口鱼 & Opsariichthys bidens & 1 & 2 & 0 & 2 & 2 & 7 \\
\hline & & 草鱼 & Ctenopharyngodon idellus & 1 & 2 & 0 & 2 & 2 & 7 \\
\hline & & 鲫 & Carassius auratus auratus & 1 & 2 & 0 & 2 & 2 & 7 \\
\hline & & 翅嘴鲌 & Culter alburnus & 0 & 2 & 0 & 2 & 2 & 6 \\
\hline & & 暗色唇鲮* & Semilabeo obscurus & 1 & 2 & 1 & 2 & 2 & 8 \\
\hline & ii 鲀科 Cobitidae & 横纹南鳅 & Schistura fasciolatus & 0 & 2 & 0 & 1 & 2 & 5 \\
\hline \multirow{6}{*}{$\begin{array}{c}\text { II 鲇形目 } \\
\text { Siluriformes }\end{array}$} & i 胡鲇科 Clariidae & 胡鲇 & Clarias batrachus & 0 & 2 & 0 & 1 & 2 & 5 \\
\hline & ii 鲿科 Bagridae & 黄颡鱼 & Pelteobagrus fulvidraco & 0 & 2 & 0 & 1 & 2 & 5 \\
\hline & & 长吻鯔 & Leiocassis longirostris & 0 & 2 & 0 & 1 & 2 & 5 \\
\hline & & 越鳠 & Mystus pluriradiatus & 0 & 2 & 0 & 1 & 2 & 5 \\
\hline & & 斑鳠 & Mystus guttatus & 0 & 2 & 0 & 1 & 2 & 5 \\
\hline & iii 鮡科 Sisoridae & 红魾* & Bagarius rutilus & 1 & 2 & 1 & 1 & 2 & 7 \\
\hline III鲈形目 & $\mathrm{i}$ 丽鱼科 Cichlidae & 罗非鱼 & Oreochromis nilotica & 1 & 2 & 0 & 2 & 0 & 5 \\
\hline Perciformes & ii 鳢科 Channidae & 乌鳢 & Ophiocephalus argus & 0 & 2 & 0 & 2 & 2 & 6 \\
\hline
\end{tabular}

*为主要过鱼对象. 
结合环评报告要求,过鱼对象的选择要优先考虑本流域所特有的地方鱼类, 即应优先国家级、省级保护 鱼类及濒危珍稀物种. 根据要求, 暗色唇鲮 (Semilabeo obscurus)、华南鲤 (Cyprinus carpio rubrofuscus) 和红魾 (Bagarius rutilus) 被确定为主要过鱼对象, 并开展了游泳能力测试 (本研究中收集到的袋唇鱼 (Paraspinibarbus hekouensis)、斑鳠(Mystus guttatus)、越鳠(Mystus pluriradiatus) 和翘嘴鲌(Culter alburnus) 数 量较少,无法满足实验统计分析要求), 每尾鱼不重复测试使用.

3 种测试鱼于 2019 年 8-9 月分多次在云南省个旧市马堵山水电站的红河 (元江流域) 干流与支流鲜水 河交汇处捕获, 总共捕获 110 尾, 其中暗色唇鲮 35 尾, 体长范围 $4.19 \sim 7.50 \mathrm{~cm}$, 华南鲤 40 尾, 体长范围 18.88 25.06 cm, 红魾 35 尾, 体长范围 33.95 38.02 cm, 捕获地点如图 1 所示. 新捕获的实验鱼在业主营地 的实验场地的便携式水池中暂养 5 天, 溶解氧不少于 $6.0 \mathrm{mg} / \mathrm{L}$, 实验前一天停止喂食, 暂养水取自红河 (元 江流域), 每天早晚各换水一次,每次换水约 $50 \%$,使用恒温机维持暂养池中的水温为 $23 \sim 25^{\circ} \mathrm{C}$.

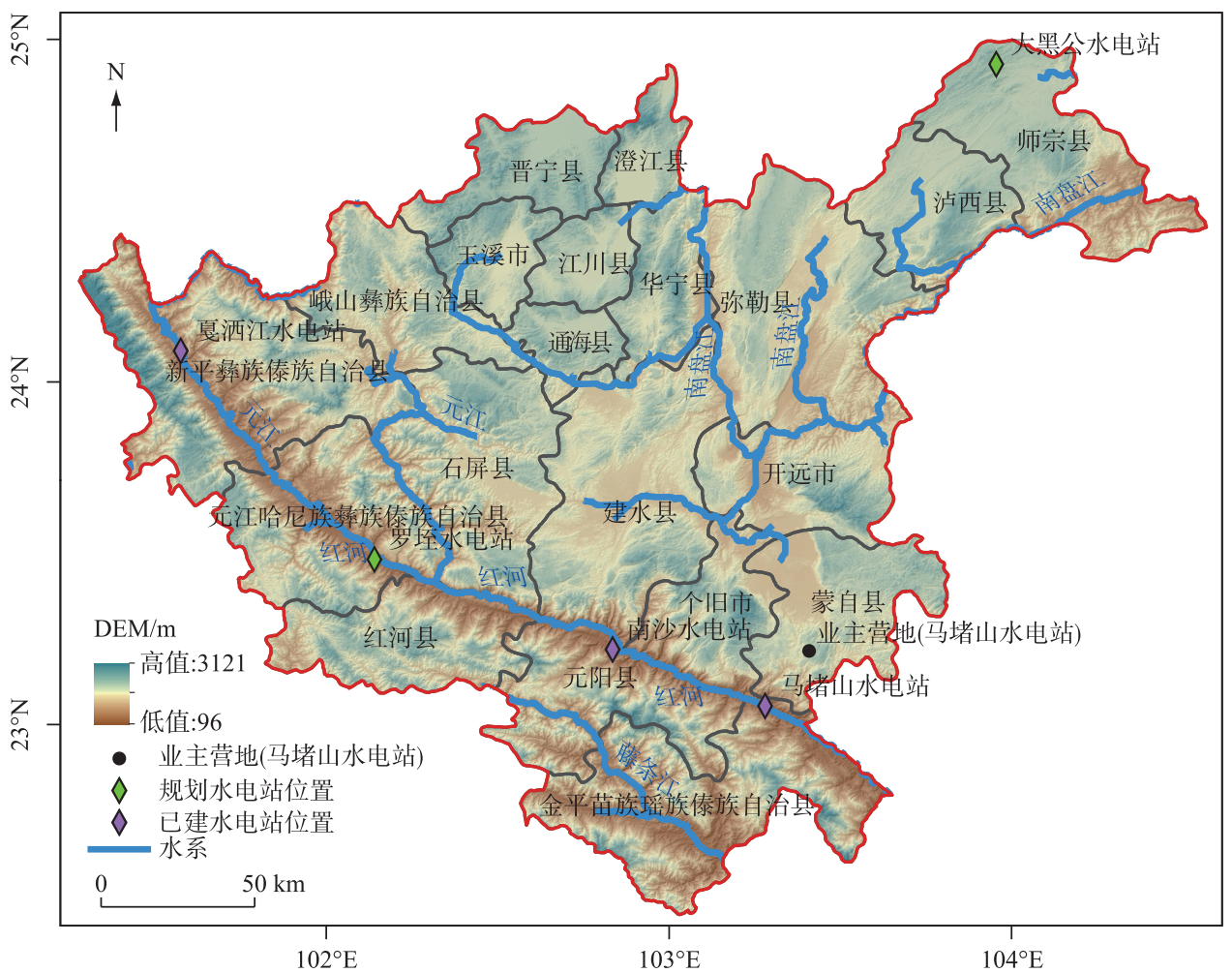

图 1 研究区域示意图

Fig.1 Sketch of the study area

\section{2 实验装置}

感应流速、临界游泳速度、突进游泳速度测试均在游泳能力呼吸测试水槽 (Loligo System SW10150) 中进 行 (图 2). 变频器控制电动机转速, 从而产生不同水流速度. 测试区上游的蜂窝状稳流装置可保证测试区域 流场均匀稳定.

\section{3 游泳能力测试}

1.3.1 感应流速 感应游泳速度 $\left(U_{\text {ind }}\right)$ 的测定采用 “递增流速法”, 将 1 尾实验鱼放置于游泳能力测试水槽 中, 静水下适应 $1 \mathrm{~h}$ 后, 先将螺旋桨反转, 当实验鱼将头摆向拦鱼网一侧均匀摆尾后, 调整使螺旋桨正转, 当 实验鱼随着水流速度缓慢增加, 出现游泳姿态摆正至头部朝向上游来水方的行为即认定该流速为实验鱼的 感应流速 ${ }^{[14-15]}$. 


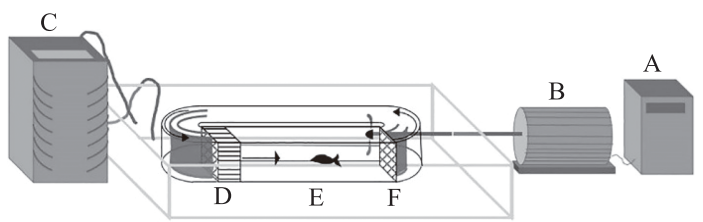

图 2 游泳能力测试水槽 ${ }^{[15,17]}$ ( $\mathrm{A}$ : 变频器; $\mathrm{B}$ : 电动机; $\mathrm{C}$ : 恒温器; D: 整流器; $\mathrm{E}$ : 游泳槽; F : 拦网)

Fig.2 Swimming ability test flume ${ }^{[15,17]}$

1.3.2 临界游泳速度 按照 “递增流速法” 进行临界游泳速度 $\left(U_{\text {crit }}\right)$ 的测定. 为了消除实验鱼的转移过程中产 生的影响, 在 $1.0 \mathrm{BL} / \mathrm{s}$ ( BL 为体长) 的水流速度下适应 $1 \mathrm{~h}$, 然后每 $20 \mathrm{~min}$ 速度增加 $1.0 \mathrm{BL} / \mathrm{s}$, 当鱼体不能抵 抗水流速度继续游泳, 其尾部贴在下游笁网且轻拍水面 $20 \mathrm{~s}$ 鱼体没有行动反应, 则视其达到力竭状态, $U_{\text {crit }}$ 测试结束 ${ }^{[21]}$.

绝对临界游泳速度 $\left(U_{\text {crit }}, \mathrm{cm} / \mathrm{s}\right)$ 的计算 ${ }^{[14,22]}$ :

$$
U_{\text {erit }}=U_{\max }+\frac{t}{\Delta t} \Delta U
$$

式中, $U_{\text {max }}$ 为实验鱼实际完成持续游泳时间的游泳速度最大值 $(\mathrm{m} / \mathrm{s}) ; \Delta t$ 为改变水流速度时间的间隔 $($ 本研 究取 $20 \mathrm{~min}$ ); $t$ 为在最高水流速度下的实验鱼游泳时间 $(\mathrm{min}) ; \Delta U$ 为水流速度的改变量 $(1.0 \mathrm{BL} / \mathrm{s})$.

相对临界游泳速度 $\left(U_{\text {crit }}^{\prime}, \mathrm{BL} / \mathrm{s}\right)$ :

$$
U_{\text {crit }}^{\prime}=\frac{U_{\text {crit }}}{B L}
$$

式中, $B L$ 为实验鱼的体长 $(\mathrm{cm})$.

当实验鱼的横截面积大于实验测试区横截面积 $20.0 \%$ 时, 会引堵塞效应, 需要对测试结果进行纠正, 本 实验所用实验鱼的横截面积均小于测试实验区横截面积的 $10.0 \%$, 故不需要纠正 ${ }^{[23]}$.

1.3.3 突进游泳速度 突进游泳速度 $\left(U_{\text {burt }}\right)$ 与临界游泳速度的测试方法和计算公式基本一致, 按照 “递增流 速法” 进行 $U_{\text {burst }}$ 的测定. 只是将流速递增时间间隔改为 $20 \mathrm{~s}$, 流速递增间隔仍为 $1.0 \mathrm{BL} / \mathrm{s}$, 此时鱼类力竭时 对应的流速为突进游泳速度 ${ }^{[14]}$.

1.3.4 最大突进上溯距离 为了分析鱼类在高流速障碍下可游多远 ${ }^{[24]}$, 本文提出了过鱼设施内的最大突进 上溯距离指标 $D_{\max }(\mathrm{m})$, 计算公式如下:

$$
D_{\max }=\left(V_{1}-V_{2}\right) \times 20
$$

式中, $V_{1}$ 为鱼在 $20 \mathrm{~s}$ 内的最大突进游泳速度 $(\mathrm{m} / \mathrm{s}) ; V_{2}$ 为过鱼设施内部的水流速度 $(\mathrm{m} / \mathrm{s})^{[25]}$.

3 种游泳能力指标测试结束后, 分别统计每条实验鱼的湿重 $(\mathrm{g}) 、$ 体长 $(\mathrm{cm})$ 、叉长 $(\mathrm{cm}) 、$ 全长 $(\mathrm{cm}) 、$ 体 高 $(\mathrm{cm})$ 、体宽 $(\mathrm{cm})$ 等形态学参数,如表 3 所示.

1.3.5 趋流率 趋流率 ( percentage of rheotaxis, Pre) 指, 在特定流速 $(V)$ 下, 通过视频观察分析, 统计实验鱼感 受水流后作出逆向反应的实验鱼的数量 $(N)$ 占进行测试的所有实验鱼总数量 $\left(N_{t}\right)$ 的百分比 ${ }^{[26-27]}$. 计算公式 如下:

$$
\text { Pre }=\left(N / N_{\mathrm{t}}\right) \times 100 \%
$$

式中, $N$ 为在特定流速条件下感受水流后作出逆向反应的实验鱼的数量, $N_{\mathrm{t}}$ 为进行测试的所有实验鱼的总 数量.

\section{4 数据处理}

实验数据用 SPSS 19.0 统计软件进行处理, 并用 Origin 2018 进行绘图. 实验鱼的感应流速、临界游泳速 度和突进游泳速度与体长的关系用 Pearson 相关性检验. 实验鱼的趋流率与水流速度的关系通过非线性回 归进行拟合. 不同体长对影响感应流速的诱导率与临界、突进游泳速度的累积疲劳率用 “生存分析曲线” 表

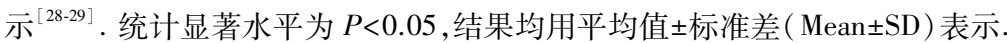


表 3 游泳能力测试实验相关参数

Tab.3 Relavant parameters of swimming ability test

\begin{tabular}{|c|c|c|c|c|c|c|c|c|}
\hline $\begin{array}{l}\text { 游泳能力 } \\
\text { 测试 }\end{array}$ & $\begin{array}{c}\text { 实验鱼 } \\
\text { 种类 }\end{array}$ & $\begin{array}{l}\text { 实验 } \\
\text { 数量 }\end{array}$ & $\begin{array}{c}\text { 湿重/ } \\
\mathrm{g}\end{array}$ & $\begin{array}{c}\text { 体长/ } \\
\mathrm{cm}\end{array}$ & $\begin{array}{c}\text { 全长/ } \\
\mathrm{cm}\end{array}$ & $\begin{array}{c}\text { 水温/ } \\
{ }^{\circ} \mathrm{C}\end{array}$ & $\begin{array}{c}\text { 肥满度/ } \\
\left(100 \mathrm{~g} / \mathrm{cm}^{3}\right)^{*}\end{array}$ & 测试时间 \\
\hline \multirow[t]{3}{*}{ 感应流速 } & 暗色唇鲮 & 12 & $7.94 \pm 0.30$ & $5.99 \pm 0.67$ & $7.99 \pm 0.76$ & $24.16 \pm 0.28$ & $3.68 \pm 0.04$ & $2019-08-25$ \\
\hline & 华南鲤 & 10 & $291.11 \pm 30.04$ & $22.58 \pm 1.71$ & $26.48 \pm 1.62$ & $23.75 \pm 0.25$ & $2.51 \pm 0.02$ & $2019-08-27$ \\
\hline & 红鮕 & 10 & $744.48 \pm 59.94$ & $41.31 \pm 2.25$ & $44.44 \pm 2.17$ & $23.51 \pm 0.21$ & $1.05 \pm 0.02$ & $2019-08-29$ \\
\hline \multirow[t]{3}{*}{ 临界游泳速度 } & 暗色唇鲮 & 12 & $8.03 \pm 0.22$ & $6.18 \pm 0.50$ & $8.20 \pm 0.57$ & $23.66 \pm 0.72$ & $3.39 \pm 0.03$ & 2019-09-01 \\
\hline & 华南鲤 & 10 & $293.52 \pm 30.03$ & $22.72 \pm 1.71$ & $26.61 \pm 1.62$ & $24.5 \pm 0.50$ & $2.48 \pm 0.04$ & 2019-09-05 \\
\hline & 红魾 & 10 & $704.71 \pm 51.20$ & $36.89 \pm 0.69$ & $40.02 \pm 0.64$ & $23.59 \pm 0.52$ & $1.40 \pm 0.02$ & $2019-09-10$ \\
\hline \multirow[t]{3}{*}{ 突进游泳速度 } & 暗色唇鲮 & 7 & $8.01 \pm 0.28$ & $6.14 \pm 0.63$ & $8.15 \pm 0.71$ & $24.33 \pm 0.44$ & $3.44 \pm 0.03$ & $2019-09-14$ \\
\hline & 华南鲤 & 10 & $292.02 \pm 27.91$ & $22.72 \pm 1.71$ & $26.52 \pm 1.57$ & $23.84 \pm 0.41$ & $2.46 \pm 0.02$ & 2019-09-17 \\
\hline & 红魾 & 10 & $705.64 \pm 51.04$ & $36.91 \pm 0.69$ & $40.03 \pm 0.64$ & $23.95 \pm 0.39$ & $1.38 \pm 0.04$ & $2019-09-20$ \\
\hline
\end{tabular}

$*$ 肥满度 $=$ 湿重 $/$ 全长 $\left(\mathrm{g} / \mathrm{cm}^{3}\right)^{[14]}$.

\section{2 结果}

\section{1 游泳能力测试结果}

2.1 .1 感应流速 暗色唇鲮的感应流速范围为 $0.06 \sim 0.07 \mathrm{~m} / \mathrm{s}$, 平均感应流速为 $(0.07 \pm 0.01) \mathrm{m} / \mathrm{s}$; 相对感应 流速范围为 $0.96 \sim 1.51 \mathrm{BL} / \mathrm{s}$, 平均值为 $(1.17 \pm 0.14) \mathrm{BL} / \mathrm{s}$. 华南鲤的感应流速范围为 $0.07 \sim 0.08 \mathrm{~m} / \mathrm{s}$, 平均感 应流速为 $(0.08 \pm 0.01) \mathrm{m} / \mathrm{s}$; 相对感应流速范围为 $0.30 \sim 0.43 \mathrm{BL} / \mathrm{s}$, 平均值为 $(0.35 \pm 0.04) \mathrm{BL} / \mathrm{s}$. 红鮕 的感应流速为 $0.09 \sim 0.10 \mathrm{~m} / \mathrm{s}$, 平均感应流速为 $(0.10 \pm 0.01) \mathrm{m} / \mathrm{s}$; 相对感应流速范围为 $0.22 \sim 0.30$ $\mathrm{BL} / \mathrm{s}$, 平均值为 $(0.24 \pm 0.02) \mathrm{BL} / \mathrm{s}$. 根据实验结果, 暗色唇鲮绝对感应流速与体长有显著性关系, 随体 长增加而增加, 拟合关系式为 $U_{\text {ind }}=0.003 B L+0.052$ $\left(R^{2}=0.69, P<0.05\right)$, 红魾和华南鲤的绝对感应流 速与体长无显著性关系 $(P>0.05)$, 但其相对感应流 速与体长有显著性关系 $(P<0.05)$, 随体长增加均减 小(图 3), 暗色唇鲮、华南鲤、红魾的相对感应流速 与体长关系可分别拟合为 $U_{\text {ind }}^{\prime}=-0.173 B L+2.206$ $\left(R^{2}=0.95, P<0.05\right) 、 U_{\text {ind }}^{\prime}=-0.019 B L+0.775\left(R^{2}=\right.$ $0.95, P<0.05)$ 和 $U_{\text {ind }}^{\prime}=-0.007 B L+0.525\left(R^{2}=0.91\right.$, $P<0.05), 3$ 种过鱼对象的感应流速大小为: 暗色唇

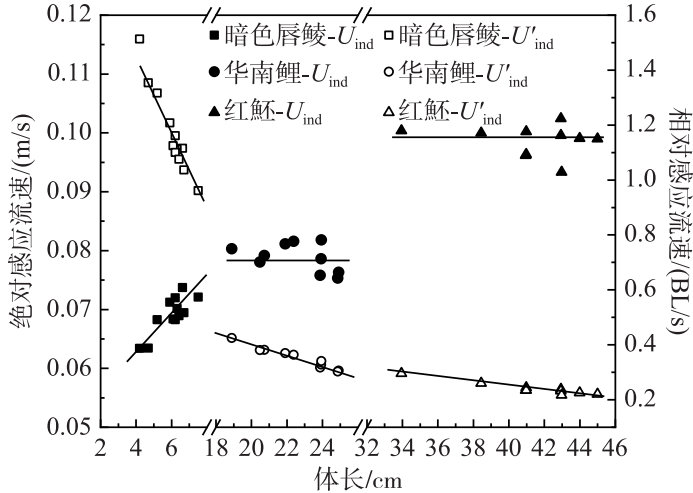

图 33 种实验鱼的感应流速与体长的关系

Fig.3 Relationships between absolute/relative induction speed and body length of three fish species 鲮 $<$ 华南鲤 $<$ 红魾.

本研究将 3 种实验鱼的趋流率 (Pre) 与水流速度 $(V)$ 进行线性拟合 (图 4), 拟合公式为 Pre $=19.80 \mathrm{~V}-$ 1.13 $\left(R^{2}=0.96, P<0.05\right)$. 结果表明, 3 种实验鱼的趋流率随着水流速度增加而增加, 当水流速度较小或者无 流速时, 趋流率较低, 当水流速度为 $0.08 \mathrm{~m} / \mathrm{s}$ 时, 趋流率为 $50 \%$, 当水流速度达到 $0.11 \mathrm{~m} / \mathrm{s}$ 时, 趋流率高 达 $95 \%$.

2.1 .2 突进游泳速度 暗色唇鲮的绝对突进游泳速度范围为 $0.77 \sim 1.11 \mathrm{~m} / \mathrm{s}$, 平均值为 $(1.01 \pm 0.10) \mathrm{m} / \mathrm{s}$; 相 对突进游泳速度范围为 $10.67 \sim 20.60 \mathrm{BL} / \mathrm{s}$, 平均值为 $(16.79 \pm 3.10) \mathrm{BL} / \mathrm{s}$. 华南鲤的绝对突进游泳速度范围 为 $1.20 \sim 1.81 \mathrm{~m} / \mathrm{s}$, 平均值为 $(1.49 \pm 0.18) \mathrm{m} / \mathrm{s}$; 相对突进游泳速度范围为 $4.80 \sim 8.78 \mathrm{BL} / \mathrm{s}$, 平均值为 $(6.62 \pm$ $1.31) \mathrm{BL} / \mathrm{s}$. 红鮕的绝对突进游泳速度范围为 $0.70 \sim 1.04 \mathrm{~m} / \mathrm{s}$, 平均值为 $(0.86 \pm 0.10) \mathrm{m} / \mathrm{s}$; 相对突进游泳速度 


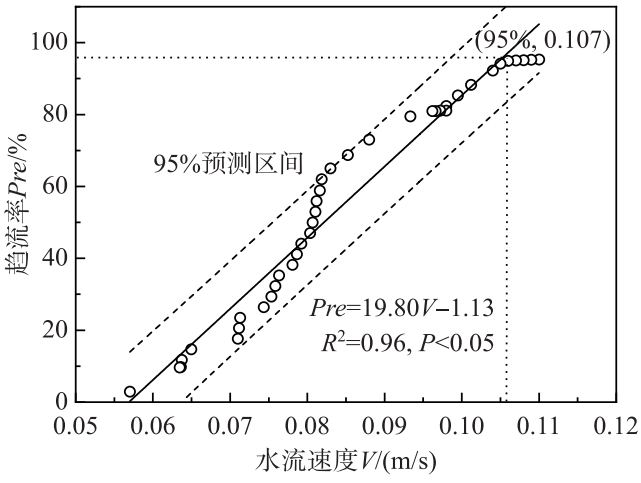

图 4 趋流率与水流速度的关系

Fig.4 Relationship between percentage of rheotaxis and flow speed

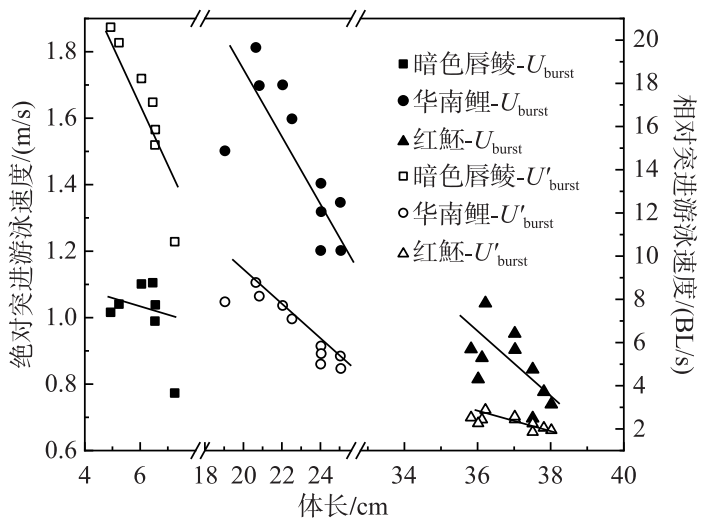

图 53 种实验鱼的突进游泳速度与体长的关系

Fig.5 Relationship between absolute/relative burst speed and body length of three fish species

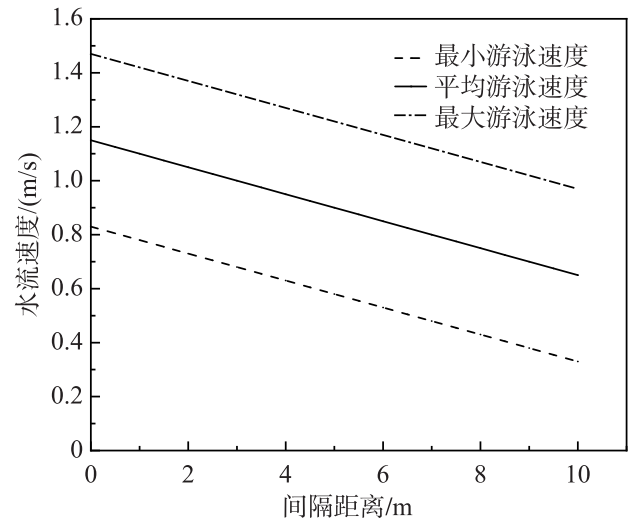

图 6 最大上溯距离与水流速度之间的关系

Fig.6 Relationship between maximum distance of ascent and the flow speed
范围为 $1.86 \sim 2.88 \mathrm{BL} / \mathrm{s}$, 平均值为 $(2.32 \pm 0.30)$ $\mathrm{BL} / \mathrm{s}$.

根据实验结果, 暗色唇鲮和红鮖的绝对突进游 泳速度与体长的关系不显著 $(P>0.05)$, 华南鲤的 绝对突进游泳速度与体长关系显著, 随着体长增加 而减小, 拟合公式为 $U_{\text {burst }}=-0.079 B L+3.27\left(R^{2}=\right.$ $0.55, P<0.05)$; 暗色唇鲮、华南鲤和红魾的相对突 进游泳速度均随体长增加而减少, 拟合公式分别为 $U_{\text {burst }}^{\prime}=-3.875 B L+40.587\left(R^{2}=0.88, P<0.05\right) 、 U_{\text {burst }}^{\prime}=$ $-0.65 B L+21.386\left(R^{2}=0.83, P<0.05\right)$ 和 $U_{\text {burst }}^{\prime}=$ $-0.27 B L+12.288\left(R^{2}=0.49, P<0.05\right), 3$ 种过鱼对 象的突进游泳速度大小为: 红魾 $<$ 暗色唇鲮 $<$ 华南鲤 (图 5).

3 种实验鱼的最小突进游泳速度为 $0.83 \mathrm{~m} / \mathrm{s}$, 平均突进游泳速度为 $1.11 \mathrm{~m} / \mathrm{s}$, 最大突进游泳速度 为 $1.47 \mathrm{~m} / \mathrm{s}$. 根据公式 (3) 可得到鱼在突进游泳速 度下的最大上溯距离与水流速度之间的关系 (图 6).

2.1.3 临界游泳速度 暗色唇鲮的绝对临界游泳速 度范围为 $0.57 \sim 0.59 \mathrm{~m} / \mathrm{s}$, 平均值为 $(0.58 \pm 0.01)$ $\mathrm{m} / \mathrm{s}$; 相对临界游泳速度范围为 $0.67 \sim 0.64 \mathrm{BL} / \mathrm{s}$, 平均值为 $(9.44 \pm 0.80) \mathrm{BL} / \mathrm{s}$. 华南鲤的绝对临界游 泳速度范围为 $0.66 \sim 0.67 \mathrm{~m} / \mathrm{s}$, 平均值为 $(0.66 \pm$ $0.01) \mathrm{m} / \mathrm{s}$; 相对临界游泳速度范围为 $2.63 \sim 3.47$ $\mathrm{BL} / \mathrm{s}$, 平均值为 $(2.92 \pm 0.22) \mathrm{BL} / \mathrm{s}$. 红鮕的绝对临 界游泳速度范围为 $0.59 \sim 0.62 \mathrm{~m} / \mathrm{s}$, 平均值为 $(0.61 \pm 0.01) \mathrm{m} / \mathrm{s}$; 相对临界游泳速度范围为 $1.59 \sim$ $1.72 \mathrm{BL} / \mathrm{s}$, 平均值为 $(1.65 \pm 0.04) \mathrm{BL} / \mathrm{s}$.

根据实验结果, 暗色唇鲮、红魾和华南鲤的绝 对临界游速与体长无显著性关系 $(P>0.05)$; 但其 相对感应流速与体长有显著性关系 $(P<0.05)$, 随 着体长增加而减小 (图 7), 暗色唇鲮、华南鲤、红魾 的相对临界游速与体长关系可分别拟合为 $U_{\text {crit }}^{\prime}=$ $20.308-1.782 B L\left(R^{2}=0.79, P<0.05\right) 、 U_{\text {crit }}^{\prime}=5.887-$ $0.131 B L\left(R^{2}=0.95, P<0.05\right)$ 和 $U_{\text {crit }}^{\prime}=3.424-$ $0.131 B L\left(R^{2}=0.71, P<0.05\right), 3$ 种过鱼对象的临界 游泳速度大小为: 暗色唇鲮<红魾<华南鲤.

\section{2 游泳能力累积疲劳率与流速关系}

通过 “生存分析”方法, 得出 3 种实验鱼在水 流中感知水流方向的累积百分比 (感应流速) 或累 积疲劳 (临界游泳速度和突进游泳速度) 百分比曲 线,结果表明,在感知水流方向的累积百分比曲线 中, 水流速度为 $0.11 \mathrm{~m} / \mathrm{s}$ 时, $95 \%$ 的鱼能感知水流 方向; 在临界游泳速度和突进游泳速度的累积疲 


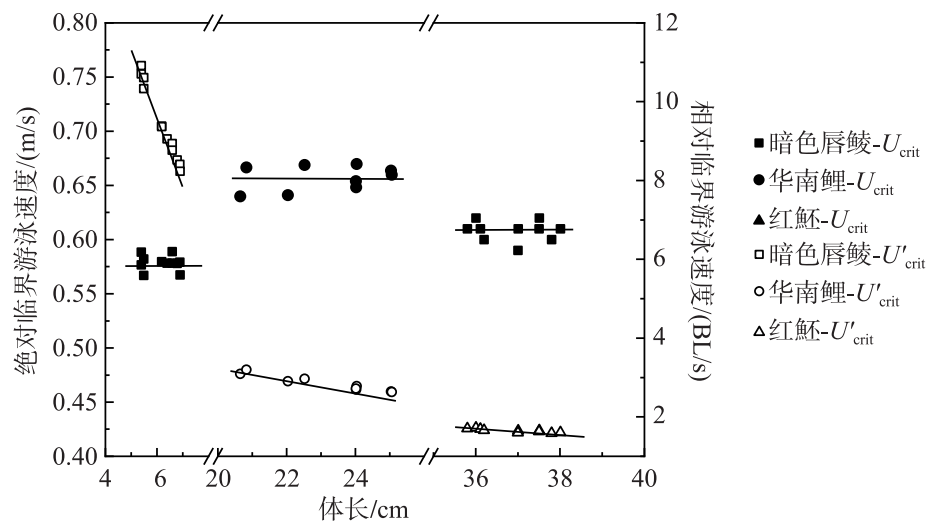

图 73 种实验鱼的临界游泳速度与体长的关系

Fig.7 Relationship between absolute/relative critical swimming speed and body length of three fish species 劳曲线中, $95 \%$ 的鱼非疲劳临界、突进游泳速度分别为 0.58 和 $1.01 \mathrm{~m} / \mathrm{s}$, 如图 8 所示.

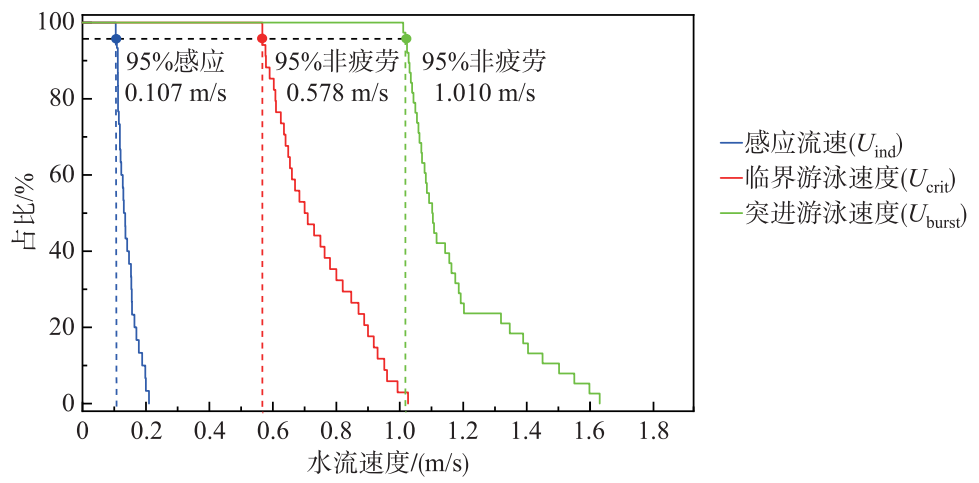

图 8 感应流速、临界游泳速度和突进游泳速度的累积疲劳率与水流流速的关系

Fig. 8 Relationship between cumulative percentages and velocity in induced flow velocity test, critical swimming speed test and burst swimming speed test

\section{3 讨论}

过鱼设施的结构和流速设计通常需要参考过鱼对象的游泳能力数据 ${ }^{[14,27]}$. 目前在元江流域有关过坝需 求的鱼类游泳能力数据处于空白, 本研究补充了元江流域 3 种典型洄游性鱼类的游泳能力, 分析了体长对 游泳能力的影响, 比较了 3 种鱼类游泳能力之间的差异, 提出了过鱼设施中不同部位的流速设计建议值, 旨 在为元江流域的过鱼设施设计提供重要参考.

\section{1 体长对游泳能力的影响}

通过构建 3 种实验鱼的体长与游泳能力的函数关系, 发现 3 种实验鱼的相对游泳能力与体长相关性更 高, 均随着体长增加而减小, 这与 Hou 等研究结果相吻合 ${ }^{[30]}$. 鱼类游泳能力受多种因素的影响, 但体长是影 响鱼类游泳能力的主要因素 ${ }^{[31-32]} .3$ 种实验鱼的相对游泳能力随体长增大而减小的主要原因可能是鱼的有 氧运动能力, 其随着鱼体长的变化而异向变化, 这意味着较小的鱼可能会比较大的鱼表现出更高的相对游 泳能力 ${ }^{[33]}$, 本研究的 3 种鱼类均表现出相同的异向变化趋势.

\section{2 游泳能力之间的比较}

不同鱼种的游泳能力存在差异, 可能与鱼的栖息生境、游泳行为和体型等因素相关. 本研究红鮕的感应 
流速相比其他两种鱼类较大, 可能原因是红魾常栖息于水流底层, 底层的水流速度较小, 对水流的敏感度较 低, 从而表现出较大的感应流速. 白艳勤等也发现 ${ }^{[34]}$, 黄颡鱼相比草鱼和鲢鱼表现出相对较低的感应流速, 与黄颡鱼定居底栖的生活习性有关. 本研究中华南鲤相比其他两种鱼表现出较强的突进游泳速度. 在进行 突进游泳速度测试时, 发现华南鲤在面对高流速时, 长时间贴壁游泳, 可能借助边壁的低流速区, 降低运动 的能量消耗, 从而产生了相对较大的突进游泳速度, 然而其他两种鱼并未发现此现象. 此外, 相比于其他两 种鱼, 华南鲤也表现出较强的临界游泳能力. 临界游泳能力与鱼的体型有关 ${ }^{[35]}$, 付翔等发现, 中华倒刺鲃比 异育银鲫的临界游泳能力高, 主要是由于中华倒刺鲃的体型更符合流线型. 本研究中通过比较 3 种实验鱼 的肥满度, 同为鲤科鱼类的华南鲤的肥满度为暗色唇鲮的 $73 \%$, 即前者的体型更符合流线型, 从而表现出较 高的临界游泳能力, 这也与付翔等研究相吻合 ${ }^{[35]}$.

\section{3 鱼类游泳能力指标在过鱼设施设计中的应用}

鱼类游泳能力主要与体长、水温和鱼种等多参数相关 ${ }^{[35-37]}$, 过鱼设施在流速设计中通常需考虑多目标 鱼种的游泳能力. 本研究考虑了过鱼设施选取过程中多鱼种和多参数的影响, 通过累计瘦劳曲线与流速的 关系, 提出 $95 \%$ 鱼类在非疲劳状态下的感应流速、临界游泳速度和突进游泳速度. Cai 等也通过此方法 ${ }^{[29]}$, 对陕西省旬阳水电站开展了过鱼设施的流速设计, 研究分析出该大坝以趐嘴鲌 (Culter alburnus)、蒙古鲌 (Culter mongolicus)、多鳞白甲鱼 (Onychostoma macrolepis) 和大眼鲘 (Siniperca kneri) 等 7 种鱼类为过鱼对象 的 $95 \%$ 鱼类在非疲劳状态下的感应流速、临界游泳速度和突进游泳速度分别为 $0.17 、 0.59$ 和 $0.94 \mathrm{~m} / \mathrm{s}$. 本研 究结果与 Cai 等研究结果相似, 说明过鱼对象虽然不同, 但在选取过鱼设施局部位置流速时具有一定的共性 需求. 本研究通过累积疲劳率曲线和鱼类游泳能力分析了元江流域过鱼设施的局部流速设计, 参考值如下. 3.3.1 过鱼设施入口流速 过鱼设施(升鱼机、鱼道、鱼闸或集鱼船)进口进行流速设计时,会利用较大的水 流吸引并帮助鱼类找到过鱼设施人口, 若人口流速过大会妨碍鱼类进人, 若流速太小, 则对鱼类的吸引力不 足, 过鱼设施人口设计流速应大于临界游泳速度且小于突进游泳速度 ${ }^{[1,38]}$. 基于本研究的累积疲劳百分比 曲线 (图 7), 为保证 $95 \%$ 的鱼类能感知并找到过鱼设施人口, 建议人口流速范围为 $0.6 \sim 1.0 \mathrm{~m} / \mathrm{s}$.

3.3.2 过鱼整体平均流速 鱼类成功通过人口后到达过鱼设施通道内部, 通道内部的流速设计过小, 鱼类将 失去趋流性, 建议过鱼设施的内部流速应大于感应流速且小于临界游泳速度 ${ }^{[17]}$. 当过鱼对象为暗色唇鲮、 华南鲤和红鮕时, 为保证 $95 \%$ 的鱼类在过鱼设施内有趋流反应, 建议过鱼设施内部整体平均流速设计范围 为 $0.1 \sim 0.6 \mathrm{~m} / \mathrm{s}$.

3.3.3 过鱼设施休息区流速在过鱼设施设计中, 为保证鱼类有足够的体能通过较长的过鱼设施, 过鱼设施 的流速设计有必要考虑鱼是否产生疲劳. 根据相关研究, 疲劳会影响鱼类的健康和生存, 如果鱼类经常或持 续高速游泳, 体内会产生大量的乳酸, 鱼类死亡率达到 $40 \%$ 以上 ${ }^{[39]}$. 故在过鱼设施内部提供鱼类低流速区 的休息场所,待鱼类基本恢复或完全恢复体能后继续上溯. 鱼类在流速过高的休息区停留会导致疲劳,但流 速过小则会导致鱼类失去方向感并长时间停留, 为使鱼类在休息区恢复体能的同时不迷失其方向感,过鱼 设施休息区域的流速建议介于过鱼对象的感应流速和临界游泳速度之间 ${ }^{[38-40]}$. 结合本研究结果, 当过鱼对 象为暗色唇鲮、华南鲤和红鮕时,建议休息区的水流速度范围为 $0.1 \sim 0.6 \mathrm{~m} / \mathrm{s}$.

3.3.4 过鱼设施出口流速 过鱼设施出口流量应比较平稳, 以便于鱼类游出过鱼设施后不影响其在上游的正 常洄游行为, 故过鱼设施的出口位置应布置在流速相对稳定的水域, 保证鱼类感应到水流, 不会迷失方

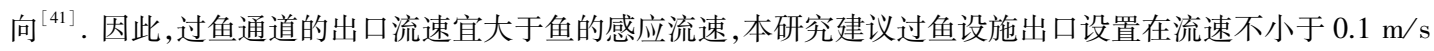
的水域.

3.3.5 过鱼设施孔口流速 过鱼设施内部的高流速区域一般是在孔口或坚缝处等. 鱼类通过过鱼设施孔口 时, 基本都是以突进游泳速度在短时间内通过 ${ }^{[42]}$. 根据图 5 , 若鱼以最大游泳速度通过鱼道内长度为 $30 \mathrm{~cm}$ 的坚缝, 为保证弱游泳能力的鱼类顺利通过, 鱼道坚缝处的流速应不超过 $0.8 \mathrm{~m} / \mathrm{s}$. 本研究以游泳能力相对 较弱的红魾的突进游泳速度为孔口或者坚缝处的最大设计流速, 建议孔口或者坚缝处的流速不宜超过 0.8 $\mathrm{m} / \mathrm{s}$. 此外, 有研究表明, 由于在靠近分界面时流速分布较低, 适当增加过鱼设施孔口边壁或底部的粗䊅度, 可供中下层游泳能力较弱的鱼类通过 ${ }^{[34]}$, 此时过鱼设施孔口的设计流速可适当增加至 $0.8 \sim 1.0 \mathrm{~m} / \mathrm{s}$.

鱼道内流态复杂, 除了流速, 影响鱼类上溯行为可能与湍动能、雷诺剪应力和涡等水力因子相关 ${ }^{[8,10]}$. 
本研究是在室内封闭水槽内进行测试, 流场比较单一, 无法真实地反映出自然流态, 具有一定的局限 性 ${ }^{[38,43]}$. 未来的工研究工作需在实际鱼道模型中进行 ${ }^{[10,44-45]}$, 可有助于理解鱼类在复杂流态下上溯洄游行 为及对水力因子的响应, 从而有利于优化过鱼设施设计,提升鱼道过鱼效果.

\section{4 参考文献}

[ 1 ] Zhou JP, Qian GL. The planning and development status of thirteen hydropower bases. Shuili Shuidian Shigong, 2011,36 (1) : 1-7. [周建平, 钱钢粮. 十三大水电基地的规划及其开发现状. 水利水电施工, 2011, 36(1): 1-7.]

[ 2 ] Barbarossa V, Schmitt RJP, Huijbregts MAJ et al. Impacts of current and future large dams on the geographic range connectivity of freshwater fish worldwide. PNAS, 2020, 117(7) : 3648-3655. DOI: 10.1073/pnas.1912776117.

[ 3 ] Tao JP, Wen JY, He D et al. Review on monitoring and evaluating of fish passage facilities for upper migration. Resources and Environment in the Yangtze Basin, 2018, 27(10): 121-133. [ 陶江平, 温静雅, 贺达等. 上行过鱼设施过鱼效果 监测研究进展. 长江流域资源与环境, 2018, 27(10): 121-133.]

[ 4 ] Jin ZJ, Shan CK, Cui L et al. Design of entrance and attraction flow of fish passage facility. Water Resources Protection, 2019, 35 (6) : 145-154. [ 金志军, 单承康, 崔否等. 过鱼设施进口及吸引流设计. 水资源保护, 2019, 35(6) : 145-154. ]

[ 5 ] Shi XT, Kynard B, Liu DF et al. Development of fish passage in China. Fisheries, 2015, 40(4) : 161-169. DOI: 10. 1080/03632415.2015.1017634.

[ 6 ] Chen QW, Zhang JY, Mo KL et al. Effects of hydropower development on aquatic eco-environment and adaptive managements. Advances in Water Science, 2020, 31(5): 793-810. [ 陈求稳, 张建云, 莫康乐等. 水电工程水生态环境效应评 价方法与调控措施. 水科学进展, 2020, 31(5): 793-810.]

[ 7 ] Lin CY, Dai HC, Shi XT et al. Investigating feasible light configurations for fish restoration: An ethological insight. Fisheries Research, 2021, 234: 105807. DOI: 10.1016/j.fishres.2020.105807.

[ 8 ] Li GN, Sun SK, Liu HT et al. Schizothorax prenanti swimming behavior in response to different flow patterns in vertical slot fishways with different slot positions. Science of the Total Environment, 2021, 754: 142142. DOI: 10.1016/j. scitotenv. 2020.142142 .

[ 9 ] Jin ZJ, Ma WZ, Zhang YN et al. Assessing the swimming ability and performance of Schizothorax oconnori to cross velocity barriers in fishway. Journal of Hydraulic Engineering, 2018, 49(4) : 512-522. [ 金志军, 马卫忠, 张袁宁等. 异齿裂腹 鱼通过鱼道内流速障碍能力及行为. 水利学报, 2018, 49(4): 512-522.]

[10] Tan JJ, Gao Z, Dai HC et al. The correlation analysis between hydraulic characteristics of vertical slot fishway and fish movement characteristics. Journal of Hydraulic Engineering, 2017, 48(8) : 924-932, 944. [谭均军, 高柱, 戴会超等. 竖缝式鱼道水力特性与鱼类运动特性相关性分析. 水利学报, 2017, 48(8)：924-932, 944.]

[11] Reidy SP, Kerr SR, Nelson JA. Aerobic and anaerobic swimming performance of individual Atlantic cod. The Journal of Experimental Biology, 2000, 203(Pt 2) : 347-357.

[12] Zhao XK, Han ZE. Experiments on the current overcoming ability of some freshwater fishes. Journal of Fisheries of China, 1980, 4(1) : 31-37. [赵希坤, 韩桢锷. 鱼类克服流速能力的实验. 水产学报, 1980, 4(1) : 31-37.]

[13] Kirk MA, Caudill CC, Syms JC et al. Context-dependent responses to turbulence for an anguilliform swimming fish, Pacific lamprey, during passage of an experimental vertical-slot weir. Ecological Engineering, 2017, 106: 296-307. DOI: 10. 1016/j.ecoleng.2017.05.046.

[14] Li ZM, Chen MX, Jin ZJ et al. Swimming ability of Schizothorax irregularis ddy in Yarkand river. Chinese Journal of Ecolo$g y, 2018,37(6)$ : 1897-1902. DOI: 10.13292/j.1000-4890.201806.005. [李志敏, 陈明曦, 金志军等. 叶尔㒸河厚 唇裂腹鱼的游泳能力. 生态学杂志, 2018, 37(6) : 1897-1902.]

[15] Wang YM, Li ZM, Tu ZY et al. Fishway design based on the swimming ability of two Schizothorax species in the Yalung River, China. Chinese Journal of Applied Ecology, 2020, 31(8) : 2785-2792. DOI: 10.13287/j.1001-9332.202008.040. [王永猛, 李志敏, 涂志英等. 基于雅砻江两种裂腹鱼游泳能力的鱼道设计. 应用生态学报, 2020, 31 (8): 2785-2792.]

[16] Ye C. Effect of flow velocity on swimming behavior and energy metabolism of Leptobotia elongates [Dissertation ]. Chongqing: Southwest University of China, 2014. [叶超. 流速对长薄鳅游泳行为及运动能量代谢的影响 [ 学位论 


\section{文]. 重庆: 西南大学, 2014.]}

[17] Cai L, Katopodis C, Johnson D et al. Case study: Targeting species and applying swimming performance data to fish lift design for the Huangdeng Dam on the upper Mekong River. Ecological Engineering, 2018, 122: 32-38. DOI: 10.1016/j. ecoleng.2018.07.023.

[18] Rouleau S, Glémet H, Magnan P. Effects of morphology on swimming performance in wild and laboratory crosses of brook trout ecotypes. Functional Ecology, 2010, 24(2) : 310-321. DOI: 10.1111/j.1365-2435.2009.01636.x.

[19] Chen ZM, Chen YR. The effects on fishes results from water exploitation a long Yuanjiang Honghe River and conservations measures. Journal of Xinyang Normal Universty, 2006, (1) : 51-55, 76. [陈自明, 陈银瑞. 元江-红河水道开发对鱼类 的影响和保护对策. 信阳师范学院学报, 2006, (1) : 51-55, 76.]

[20] Schletterer M, Reindl R, Thonhauser S. Options for re-establishing river continuity, with an emphasis on the special solution "fish lift": Examples from Austria. Revista Eletrônica De Gestão e Tecnologias Ambientais, 2016, 4(1): 109. DOI: 10.9771/gesta.v4i1.16954.

[21] Fang M, Cai L, Gao Y et al. Effect of temperature on swimming capability and oxygen consumption of juvenile Hypophthalmichthys molitrix. Journal of Hydroecology, 2013, 34(3) : 49-53. DOI: 10.15928/j.1674-3075.2013.03.010. [房敏, 蔡 露, 高勇等. 温度对鲢幼鱼游泳能力及耗氧率的影响. 水生态学杂志, 2013, 34(3): 49-53.]

[22] Brett JR. The respiratory metabolism and swimming performance of young sockeye salmon. Journal of the Fisheries Research Board of Canada, 1964, 21(5) : 1183-1226. DOI: 10.1139/f64-103.

[23] Jain KE, Farrell AP. Influence of seasonal temperature on the repeat swimming performance of rainbow trout Oncorhynchus mykiss. The Journal of Experimental Biology, 2003 , 206(20) : 3569-3579. DOI: 10.1242/jeb.00588.

[24] Zheng JX, Han DJ, Hu WB et al. Fish swimming performance related to fishway design. Journal of Hydroecology, 2010, 31 (5) : 104-110. DOI: 10.15928/j.1674-3075.2010.05.023. [郑金秀, 韩德举, 胡望斌等. 与鱼道设计相关的鱼类游 泳行为研究. 水生态学杂志, 2010, 31(5): 104-110.]

[25] Peake S, Wh Beamish F, McKinley RS et al. Relating swimming performance of lake sturgeon, Acipenser fulvescens, to fishway design. Canadian Journal of Fisheries and Aquatic Sciences, 1997, 54(6) : 1361-1366. DOI: 10.1139/f97-039.

[26] Zhang S, Chen Y. Preliminary study on the rheotaxis of juvenile Sebastodes fuscescens. Journal of Shanghai Fisheries University, 2005, 14(3): 282-287. [张硕, 陈勇. 黑鲪幼鱼趋流性的初步研究. 上海水产大学学报, 2005, 14(3): 282-287.]

[27] Cai L, Wang WY, Wang HL et al. Response of induced flow speed to fish body length and its application in flow design of fish passage facilities. Transactions of the Chinese Society of Agricultural Engineering, 2018, 34(2) : 176-181. [蔡露, 王 伟营, 王海龙等. 鱼感应流速对体长的响应及在过鱼设施流速设计中的应用. 农业工程学报, 2018, 34(2): 176-181.]

[28] de Andrade e Santos H, de Faria Viana EM, Pompeu PS et al. Optimal swim speeds by respirometer: An analysis of three neotropical species. Neotropical Ichthyology, 2012, 10(4) : 805-811. DOI: 10.1590/s1679-62252012000400013.

[29] Cai L, Chen JH, Johnson D et al. Effect of body length on swimming capability and vertical slot fishway design. Global Ecology and Conservation, 2020, 22: e00990. DOI: 10.1016/j.gecco.2020.e00990.

[30] Hou Y, Cai L, Wang X et al. Swimming performance of 12 Schizothoracinae species from five rivers. Journal of Fish Biology, 2018, 92(6) : 2022-2028. DOI: 10.1111/jfb.13632.

[31] Hammer C. Fatigue and exercise tests with fish. Comparative Biochemistry and Physiology Part A: Physiology, 1995,112 (1) : 1-20. DOI: 10.1016/0300-9629(95)00060-K.

[32] Plaut I. Critical swimming speed: Its ecological relevance. Comparative Biochemistry and Physiology Part A: Molecular \& Integrative Physiology, 2001, 131(1) : 41-50. DOI: 10.1016/S1095-6433(01)00462-7.

[33] Ke SF, Li ZM, Jiang ZW et al. Effect of a vertical half cylinder on swimming of silver carp, Hypophthalmichthys molitrix: Implications for microhabitat restoration and fishway design. River Research and Applications, 2019, 35(4) : 436-441.

[34] Bai YQ, Lu B, Luo J et al. Induction velocity of juvenile grass carp, silver carp, and darkbarbel catfish. Chinese Journal of Ecology, 2013, 32(8) : 2085-2089. DOI: 10.13292/j.1000-4890.2013.0412. [白艳勤, 路波, 罗佳等. 草鱼、鲢和 瓦氏黄颡鱼幼鱼感应流速的比较. 生态学杂志, 2013, 32(8) : 2085-2089.]

[35] Fu X, Fu C, Fu SJ. Comparison of swimming ability among five freshwater fish species. Chinese Journal of Ecology, 2020, 39(5) : 1629-1635. DOI: 10.13292/j.1000-4890.202005.027. [付翔, 付成, 付世建. 五种淡水鱼类幼鱼游泳能力的 
比较. 生态学杂志, 2020, 39(5): 1629-1635.]

[36] Mo WJ, Wang CF, Liu DF et al. Study on burst swimming speeds of two typical releasing fish from Beipan River. South China Fisheries Science, 2014, 10(3) : 9-14. [莫伟均, 王从锋, 刘德富等. 北盘江 2 种典型增殖放流鱼类突进游速 研究. 南方水产科学, 2014, 10(3) : 9-14.]

[37] Xiong F, Wang CF, Liu DF et al. Comparative study of burst swimming speed of black carp, grass carp, silver carp and bighead carp from Songhua River. Ecological Science, 2014, 33(2): 339-343. [熊锋, 王从锋, 刘德富等. 松花江流域 青鱼、草鱼、鲢及鳙突进游速比较研究. 生态科学, 2014, 33(2):339-343.]

[38] Lei QS, Tu ZY, Shi XL et al. Swimming ability of Diptychus maculatus Steindachner in the Muzati River for fishway design. Journal of Fisheries of China , 2020, 44(10) : 1718-1727. [雷青松, 涂志英, 石迅雷等. 应用于鱼道设计的新疆 木扎提河斑重唇鱼的游泳能力测试. 水产学报, 2020, 44(10): 1718-1727.]

[39] Roscoe DW, Hinch SG, Cooke SJ et al. Fishway passage and post-passage mortality of up-river migrating sockeye salmon in the Seton River, British Columbia. River Research and Applications, 2011, 27(6) : 693-705. DOI: 10.1002/rra.1384.

[40] Katopodis C, Cai L, Johnson D. Sturgeon survival: The role of swimming performance and fish passage research. Fisheries Research, 2019, 212: 162-171. DOI: 10.1016/j.fishres.2018.12.027.

[41] Ding SB, Shi JY, Huang B et al. Swimming capability of six typical fish species from the lower Dadu river. Journal of Hydroecology, 2020, 41(1) : 46-52. DOI: 10.15928/j.1674-3075.2020.01.007. [丁少波, 施家月, 黄滨等. 大渡河下 游典型鱼类的游泳能力测试. 水生态学杂志, 2020, 41(1) : 46-52.]

[42] Tummers JS, Kerr JR, O'Brien P et al. Enhancing the upstream passage of river lamprey at a microhydropower installation using horizontally-mounted studded tiles. Ecological Engineering, 2018, 125: 87-97. DOI: 10.1016/j. ecoleng. 2018. 10.015 .

[43] Tudorache C, Viaenen P, Blust R et al. Longer flumes increase critical swimming speeds by increasing burst-glide swimming duration in carp Cyprinus carpio, L. Journal of Fish Biology, 2007, 71 (6) : 1630-1638. DOI: 10.1111/j.10958649.2007.01620.x.

[44] Tan JJ, Gao Z, Dai HC et al. Effects of turbulence and velocity on the movement behaviour of bighead carp (Hypophthalmichthys nobilis) in an experimental vertical slot fishway. Ecological Engineering, 2019, 127: 363-374. DOI: 10.1016/j. ecoleng.2018.12.002.

[45] Li GN, Sun SK, Zhang C et al. Evaluation of flow patterns in vertical slot fishways with different slot positions based on a comparison passage experiment for juvenile grass carp. Ecological Engineering, 2019, 133: 148-159. DOI: 10.1016/j. ecoleng.2019.04.008. 